\title{
Noncriterial recollection influences metacognitive monitoring and control processes
}

\author{
Gene A. Brewer and Richard L. Marsh \\ University of Georgia, Athens, GA, USA \\ Arlo Clark-Foos \\ University of Michigan, Dearborn, MI, USA \\ Joseph T. Meeks \\ Southern Illinois University Edwardsville, Edwardsville, IL, USA
}

\begin{abstract}
When retrieving information from memory, temporarily irrelevant material may influence future retrieval endeavours. According to an accessibility account, the amount and intensity of this information can be used to predict the availability of related material. A dual-source paradigm was used to investigate whether information that was not relevant (i.e., noncriterial recollection) to the current memory search would influence metacognitive judgements about a relevant, criterial dimension. In two experiments, participants gave higher feelings-of-knowing judgements for a weakly encoded source dimension when they could subsequently recall the other source dimension later. Furthermore, the influence of the noncriterial information appeared to be driven more so by the subjective state of remembering rather than knowing. Thus, strong memorial information that is temporarily irrelevant influences behaviour.
\end{abstract}

Keywords: Recollection; Metacognition; Source memory.

The products of retrieval from long-term memory serve many purposes for everyday functioning. Free recall, cued recall, and recognition tasks are designed to investigate how information is retrieved from memory and subsequently evaluated. In these tasks, people rely on the reactivation of various qualitative characteristics from previous experiences to make decisions about their memories or, alternatively, to continue attempting to retrieve information from memory. For example, Johnson, Hashtroudi, and Lindsay (1993) described how people can rely on multiple decision processes to weight the average amounts of qualitative characteristics that are retrieved in service of monitoring the original source of those representations. These qualitative characteristics appear to influence participants' phenomenological experience of a studied episode in multiple ways (e.g., Mather, Henkel, \& Johnson, 1997). Moreover, these characteristics can influence participants' subjective reports of remembering and knowing (Hicks, Marsh, \& Ritschel, 2002; Wais, Mickes, $\&$ Wixted, 2008). The current study examined how associated qualitative characteristics, which

Correspondence should be addressed to Gene A. Brewer, Department of Psychology, University of Georgia, Athens, GA 30602-3013, USA. E-mail: gabrewer@uga.edu

We thank Greg Spillers, Asher Koriat, and Colleen Parks for their comments on a previous draft of this manuscript. Also, we would like to thank Hollis Johnson and Michael Hopkins for their assistance in collecting the data.

\section{6}

(C) 2010 The Experimental Psychology Society

http://www.psypress.com/qjep

DOI:10.1080/17470210903551638 
were not criterial to an ongoing memory decision, might influence participants' metacognitive predictions about the future discriminability of a currently unretrievable source dimension (e.g., feeling-of-knowing, FOK; Hart, 1965).

Retrieval of information often brings to mind additional information that may not be directly relevant to the mnemonic task at hand. Such information has been labelled noncriterial recollection by Yonelinas and Jacoby (1996; see also Toth \& Parks, 2006). In noncriterial recollection paradigms, participants encode information from two source dimensions simultaneously (e.g., gender and spatial dimensions). Typically, one source dimension is deeply encoded whereas the other is weakly encoded. At retrieval, participants are given two different source tests, one for each dimension. The results from these types of experimental design have been used to investigate the independence assumption of the processdissociation procedure (Mulligan \& Hirshman, 1997; Parks, 2007). However, the present study does not attempt to speak to the debate over independence and process purity in dual-process theories of memory. Rather, the noncriterialrecollection paradigm was used here to investigate associative memory and its relationship to metacognitive monitoring and control processes.

To achieve this goal we used the dual-source paradigm to investigate participants' FOK judgements about a weakly encoded source dimension. FOKs are metamemorial predictions about the likelihood that criterial information to a failed memory query actually resides in memory (availability) and could be retrieved at a later point in time (accessibility). These predictions can be made relatively quickly reflecting decisions based on the familiarity of the test probe, or on the products of retrieval (Koriat \& Levy-Sadot, 2001; Reder \& Ritter, 1992). According to the accessibility account of FOK judgements, participants rely on the amount and intensity of partial information to guide their predictions of later successful retrieval or recognition (Koriat, 1993). An important assumption of the accessibility model is that the partial information that influences the FOK prediction does not have to be correct in order to influence the judgement. Consequently, another premise of our study was to test the assumption that all partial information, both correct and incorrect, should influence participants' FOK judgements.

In the following three experiments, participants were asked whether they could remember a weakly encoded source attribute, and when they could not they predicted their ability to recognize this attribute if it was re-presented to them. Subsequent to this source test on the weak attribute, participants' memory was tested for the deeply encoded attribute of these same items. Our key question of interest was whether metacognitive predictions (FOK) would be greater when participants had irrelevant information available (i.e., noncriterial recollection) than when they did not. The rationale was that additional information about a memory trace, even if it is noncriterial for the current memory judgement, would nevertheless influence confidence in one's ability to answer the criterial question at a later point in time. Accordingly, memory for a temporarily irrelevant dimension should influence participant's FOK predictions. In the current experiments, a dualsource paradigm was used, which was similar to Yonelinas and Jacoby's (1996) noncriterial recollection paradigm. In Experiment 1A, we expected that stronger memory for the irrelevant source dimension would lead to higher FOK predictions. In Experiment 1B, we expected that incorrect noncriterial information about an irrelevant source dimension would engender higher FOK judgements than no information at all. In Experiment 2, we replicated Experiments 1A and $1 \mathrm{~B}$, but we also asked participants during the subsequent test to give a remember versus a know response to the deeply encoded source dimension.

\section{EXPERIMENTS 1A AND 1B}

\section{Method}

\section{Participants}

A total of 55 undergraduates were recruited from the University of Georgia in exchange for credit toward a research appreciation requirement. A 
total of 30 people participated in Experiment 1A, and 25 people participated in Experiment 1B. Each person was tested individually in sessions that lasted approximately 20-30 min.

\section{Materials and procedure}

A total of 80 pictures were randomly chosen from the Snodgrass and Vanderwart (1980) norms. The software randomly picked either a coloured or a line drawing version of each picture with the only constraint being that half of the pictures appeared in each format. In addition, a male and a female speaker had prerecorded verbal labels for each picture in the set, and these stimuli were chosen to match each picture. The gender of the speaker was also randomly chosen anew for each participant with the only constraint being that half of the colour pictures and half of the line drawings were spoken by a different gender. The stimuli were presented with each picture appearing on the screen accompanied by a speaker's voice providing the verbal label.

Participants were instructed that they were going to study pictures for a later memory test. In addition, a critical aspect of the procedure was that they were informed to pay attention to whether the picture was presented in colour or as a line drawing. As such, the participants believed that the speaker's voice was simply providing a verbal label and that the gender of the speaker was irrelevant for the later memory test. Thus, the picture dimension was encoded in more detail than the gender dimension. The 80 pictures appeared on the screen for 4 seconds each with the voice label appearing simultaneously. Subsequent to this encoding phase a first test phase ensued where people were asked to indicate whether or not a male or female spoke each word. During this test, each verbal label was randomly presented on the screen, and the participants attempted to discriminate the gender of the speaker of that label heard at learning. They pressed one of two corresponding keys on the keyboard to do so. However, we provided a third response option of "don't know" with the added instructions that we knew this was a surprise test. Accordingly, we asked them to be confident in their gender judgement or otherwise use the "don't know" response option. These instructions insured that we would obtain many "don't know" responses. After making each "don't know" response, the participants had to rate their likelihood (i.e., FOK) of recognizing the gender of the speaker of the verbal label if the word was spoken again. Participants made their FOK rating on a scale from 1 (not likely to recognize the speaker) to 5 (very likely to recognize the speaker). The gender test always preceded the picture test to reduce the effects of testing on the strong dimension influencing metacognitive judgements about the weak dimension. ${ }^{1}$ After making a decision about all 80 words, a second test phase asked participants to complete a discrimination test again based this time on their memory for the picture modality. In Experiment 1A, all participants judged whether the pictures referred to by their labels were originally presented in colour or as a line drawing. In Experiment 1B, all participants made the same responses to pictures as in Experiment 1A but they were also asked to respond "don't know" whenever they were not confident enough to make a discrimination about the picture dimension. The extra "don't know" judgement for the picture dimension was the only procedural difference between Experiments $1 \mathrm{~A}$ and $1 \mathrm{~B} .^{2}$ The addition of a "don't know" response for the picture test in Experiment 1B allowed for a stronger test of the accessibility model by allowing FOK judgements associated with correct and incorrect noncriterial information to be compared with FOKs made in the absence of any noncriterial information (i.e., when participants claimed "don't know" to the subsequent picture discrimination). To be clear, no new items were included

\footnotetext{
${ }^{1}$ There are other procedural variants in the order of testing that still remain to be examined including intermixing judgements in a single test or testing the strong dimension before taking metacognitive judgements about the weak dimension.

${ }^{2}$ We thank Asher Koriat for suggesting this procedural variant on Experiment $1 \mathrm{~A}$.
} 
in either test, and all stimuli were randomized anew for each phase and participant. Participants were given as much time as they needed for each response, and all reaction times were recorded. All reaction times exceeding 2.5 standard deviations of their overall mean were disregarded in the following analyses.

\section{Results}

\section{Experiment $1 \mathrm{~A}$}

In the initial gender test, $58 \%$ of the participant's responses were "don't know". In the picture discrimination test, $74 \%$ of the pictures were correctly discriminated. These results suggest that participants paid especially close attention to the picture dimension and that they were following our instructions to only make highly confident discrimination judgements in the initial gender test. Next, we created a measure that reflected participant's average confidence in being able to correctly recognize the speaker of the verbal label if it were represented to them later (i.e., their FOK). Average FOK ratings were conditionalized on later picture discrimination performance (i.e., correct or incorrect source memory for the picture during the second test). FOKs were higher when later memory for the picture dimension was accurate than when it was inaccurate $(M$ $=2.79$ and $M=2.55$, respectively), $t(29)=4.81$, $p<.001, d=0.97$. A similar comparison based on participants' reaction time to say “don't know" during the first test demonstrated that participants were slower to say "don't know" when they could later accurately discriminate the picture dimension $(M=2,712.25 \mathrm{~ms}$ and $M=$ 2,411.63 ms, respectively), $t(29)=2.49, p<.05$, $d=0.46$.

\section{Experiment $1 B$}

The results from Experiment $1 \mathrm{~B}$ were generally in accordance with those reported for Experiment 1A. In the initial gender test, 62\% of the participant's responses were "don't know". In the picture discrimination, $80 \%$ of the pictures were correctly discriminated. These results suggest that participants paid especially close attention to the picture dimension and were following our instructions to only make highly confident discrimination judgements in the initial gender test. FOKs were higher when later memory for the picture dimension was accurate than when it was inaccurate, and, as predicted by the accessibility model, FOKs were lowest when participants reported having no memory for the picture dimension $(M=2.75$, $M=2.44$, and $M=2.24$, respectively), $F(2$, $48)=8.67, p<.01, \eta^{2}=.28$. The average FOK prediction differed across each level of memory for the noncriterial dimension, smallest $t(24)=1.99, p=.05$. There was also a significant effect of noncriterial information on the participant's reaction time to say "don't know" during the first test, $F(2,48)=4.51, p<.05$, $\eta^{2}=.16$. The average reaction time in the first test did not differ based on correct $(M=$ $3,100.44 \mathrm{~ms})$ or incorrect $(M=3082.92 \mathrm{~ms})$ responding in the second test, $t(24)<1$. Average reaction time to respond "don't know" in the first test was consistently faster when participants also responded "don't know" in the subsequent picture test $(M=2,491.00)$, smallest $t(24)=2.45, p<.05$.

\section{Discussion}

The results from Experiments $1 \mathrm{~A}$ and $1 \mathrm{~B}$ are consistent with the hypothesis that associated information that is independent of a memory task nevertheless influences decisions about later accessibility. Furthermore, Experiment 1B provides supporting evidence for Koriat's (1993) accessibility model in that even erroneous noncriterial information increases participants' FOK judgements. Parks (2007) demonstrated that noncriterial recollection resembles subjective remembering more so than subjective familiarity. In Experiment 2, participants made a subjective judgement about the picture dimension in order to clarify whether the subjective awareness of remembering, knowing, or both are responsible for the observed difference in FOK predictions in Experiments $1 \mathrm{~A}$ and $1 \mathrm{~B}$. 


\section{EXPERIMENT 2}

\section{Method}

\section{Participants}

A total of 30 undergraduates from the University of Georgia volunteered in exchange for credit toward a research appreciation requirement. Each person was tested in sessions that lasted approximately 20-30 $\mathrm{min}$.

\section{Materials and procedure}

The same materials and procedure as those in Experiment $1 \mathrm{~A}$ were used in this experiment with the only difference being that the testing procedure for the picture dimension was augmented with an additional decision. After making each picture judgement in the final test phase, we asked the participants whether they had recollective experience of seeing the picture (i.e., do you remember seeing the picture?) or whether they were basing their judgement on familiarity (i.e., was your decision based on familiarity?). ${ }^{3}$ The instructions were modelled after our many uses of the remember-know paradigm (Hicks \& Marsh, 1999).

\section{Results and discussion}

The results were analysed in a similar fashion to that of Experiments $1 \mathrm{~A}$ and $1 \mathrm{~B}$. In the initial gender test, $62 \%$ of the participant's responses were "don't know". In the picture discrimination test, $74 \%$ of the pictures were correctly discriminated. These results suggest that participants paid especially close attention to the picture dimension and were following our instructions to only make highly confident discrimination judgements in the initial gender test. Additionally, accurate picture memory was associated with higher FOK judgements when people could not earlier discriminate the gender dimension $(M=2.40$ vs. $M=2.07), \quad t(29)=3.35, \quad p<.01, \quad d=0.61$. Unlike Experiment 1, however, participants did not take longer to respond with a "don't know" response when they later were able to discriminate the picture dimension $(M=2,405.38$ vs. $M=$ 2,446.01), $t(29)=0.37, n s$.

To further analyse performance on the picture discrimination test, we conditionalized FOK ratings on the subjective reports of remembering and knowing. Participants' FOK predictions were greater when they reported remembering the picture dimension than when it was reported as being familiar $(M=2.50$ and $M=2.23$, respectively), $t(29)=2.82, p<.01, d=0.52$. Reaction times to make a "don't know" response were slower when conditionalized on the subjective reports of remembering than when conditionalized on knowing $(M=2,745.35$ and $M=2,331.52$, respectively), $\quad t(29)=2.05, \quad p<.05, \quad d=0.42$. Thus, participants took longer to make their "don't know" response when they had stronger subjective awareness of the picture dimension (i.e., remember), which was noncriterial to their current judgement. Subsequent to their "don't know" response, participants also made greater FOK predictions when they later claimed that they remembered seeing the picture in colour or as a line drawing, which replicates the influence of noncriterial information on current performance.

\section{GENERAL DISCUSSION}

Taken together, these results suggest that characteristics that are noncriterial to a current memory decision can influence metacognitive judgements about other characteristics of that memory. Specifically, Experiments $1 \mathrm{~A}$ and $1 \mathrm{~B}$ demonstrated that accurate (and inaccurate) noncriterial recollection of the deeply encoded source dimension was associated with participant's confidence in the future discriminability of the weakly encoded source dimension. Experiment 2 both replicated and extended these findings by demonstrating that the subjective experience of

\footnotetext{
${ }^{3}$ Note, we are agnostic as to whether participants' remember and know responses in the current study map clearly onto the underlying cognitive processes of recollection and familiarity. For simplicity, we assume in the current study that remember and know responses should be conceptualized as representing purely subjective states of awareness.
} 
remembering noncriterial details had a greater influence on FOK judgements than when this same information only seemed familiar. These results are informative insofar as they speak to the nature of associative retrieval of sourcespecifying characteristics. Noncriterial recollection, which can be either correct or erroneous in nature, will nevertheless influence how people search for associated information in memory.

Recent work by Starns and Hicks $(2005,2008)$ suggests that source dimensions are retrieved independently. The results from Experiment 2 suggest that subjectively remembering deeply encoded characteristics of the original presentation occur given the strong association to the item probe (Dewhurst \& Parry, 2000; Yonelinas \& Jacoby, 1996). Therefore, when these strong characteristics come to mind, they not only influence participant's metacognitive judgements of FOK for the weakly encoded dimension, but they also seem to influence the amount of time that participants are willing to search for such information before they ultimately conclude that they "don't know". Thus, information in memory can be indirectly cued, and when this happens, that information will influence what type of retrieval strategies people adopt. All information that comes to mind during a memory search should influence FOK predictions (Koriat \& Levy-Sadot, 2001), but the results from Experiment 2 demonstrated that not all types of subjective experience influenced participant's FOKs in the same manner. Specifically, participants gave higher FOK judgements when they subsequently reported remembering the noncriterial source dimension (Parks, 2007). This effect is partial evidence in favour of Starns and Hicks's general point that source dimensions can be cued independently, but obviously there are clear cases of dependence depending on how memory performance is defined. Therefore, the current results extend Starns and Hicks's findings by demonstrating that the source characteristics that do come to mind, and are independent of the currently sought after characteristics, will nevertheless have an effect of biasing metacognitive monitoring (FOK) and control (search termination) processes.
A plausible alternative conceptualization to the data at hand would be that participants differed in their levels of encoding for some trials as compared with others. This hypothesis predicts a high correlation between accurate picture discrimination and FOK judgements to the unknown gender dimension. Although the current data do not unequivocally rule out this interpretation, they nevertheless point to an important role of noncriterial information in predicting FOK judgements both when that information is accurate and even when it is inaccurate (see Experiment 1B). These results demonstrate that even information that is incorrectly represented still influences metacognitive monitoring processes above and beyond that found when participants reported having no additional information. As demonstrated in Experiment 2, the subjective awareness of remembering something specific about the original encoding experience also influenced participants' metacognitive monitoring and control processes more so than familiarity. Together, the results from these experiments suggest that subjective awareness has a potent influence on metacognitive judgements, even when the subjective awareness is incorrect. Thus, it remains an open question whether a trace strength hypothesis can truly account for the present results.

Our overarching goal was to examine how noncriterial information influences peoples' metacognitive monitoring and control processes. We investigated whether irrelevant information that was part of a memory trace might influence aspects of search and decisions about a temporarily relevant (i.e., criterial) source dimension. Based on our findings, noncriterial information comes to mind, either in total or as partial information, and affects performance on the criterial judgement being made. These experiments do not tease apart the exact locus of such an influence, but the effects reported here do not necessarily represent an explicit retrieval strategy for the noncriterial dimension. An important future direction will be to determine how various levels or amounts of noncriterial information influence FOK judgements (i.e., via correlating the frequency of concurrence between two source dimensions). In the current study, 
noncriterial recollection was intrinsically manipulated but future studies should artificially create differences in noncriterial recollection via independent manipulations. These manipulations will hopefully serve to establish a stronger relationship with metacognitive judgements. ${ }^{4}$ Nevertheless, the present data demonstrate that noncriterial information affects the degree to which people search their memory. In conclusion, noncriterial recollection, as labelled in the field, is quite consequential to memory performance in a variety of different ways that require serious further scrutiny with both behavioural and psychophysiological techniques.

Original manuscript received 5 February 2009 Accepted revision received 6 November 2009 First published online 1 March 2010

\section{REFERENCES}

Dewhurst, S. A., \& Parry, L. A. (2000). Emotionality, distinctiveness, and recollective experience. European Journal of Cognitive Psychology, 12, 541-551.

Hart, J. T. (1965). Memory and the feeling-of-knowing experience. Journal of Educational Psychology, 56, 208-216.

Hicks, J. L., \& Marsh, R. L. (1999). Remember-know judgments can depend on how the question is asked. Psychonomic Bulletin E Review, 6, 117-122.

Hicks, J. L., Marsh, R. L., \& Ritschel, L. (2002). The role of recollection and partial information in source monitoring. Journal of Experimental Psychology: Learning, Memory, and Cognition, 28, 503-508.

Johnson, M. K., Hashtroudi, S., \& Lindsay, D. S. (1993). Source monitoring. Psychological Bulletin, 114, 3-28.

Koriat, A. (1993). How do we know that we know? The accessibility model of the feeling of knowing. Psychological Review, 100, 609-639.

Koriat, A., \& Levy-Sadot, R. (2001). The combined contributions of the cue familiarity and accessibility heuristics to feeling of knowing. Journal of Experimental Psychology: Learning, Memory and Cognition, 27, 34-53.

Mather, M., Henkel, L. A., \& Johnson, M. K. (1997). Evaluating characteristics of false memories: Remember/know judgments and memory characteristics questionnaire compared. Memory \& Cognition, 25, 826-837.

Mulligan, N. W., \& Hirshman, E. (1997). Measuring the bases of recognition memory: An investigation of the process dissociation framework. Journal of Experimental Psychology: Learning Memory and Cognition, 23, 280-304.

Parks, C. M. (2007). The role of noncriterial recollection in estimating recollection and familiarity. Journal of Memory and Language, 57, 81-100.

Reder, L. M., \& Ritter, F. E. (1992). What determines initial feeling of knowing? Familiarity with question terms, not with the answer. Journal of Experimental Psychology: Learning, Memory and Cognition, 18, 435-451.

Snodgrass, J. G., \& Vanderwart, M. (1980). A standardized set of 260 pictures: Norms for name agreement, image agreement, familiarity, and visual complexity. Journal of Experimental Psychology: Human Learning and Memory, 6, 174-215.

Starns, J. J., \& Hicks, J. L. (2005). Source dimensions are retrieved independently in multidimensional monitoring tasks. Journal of Experimental Psychology: Learning, Memory, \& Cognition, 31, 1213-1220.

Starns, J. J., \& Hicks, J. L. (2008). Context attributes in memory are bound to item information, but not to one another. Psychonomic Bulletin \& Review, 15, 309-314.

Toth, J. P., \& Parks, C. M. (2006). Effects of age on estimated familiarity in the process dissociation procedure: The role of noncriterial recollection. Memory E Cognition, 34, 527-537.

Wais, P. E., Mickes, L., \& Wixted, J. T. (2008). Remember/know judgments probe degrees of recollection. Journal of Cognitive Neuroscience, 20, 400-405.

Yonelinas, A. P., \& Jacoby, L. L. (1996). Noncriterial recollection: Familiarity as automatic, irrelevant recollection. Consciousness E Cognition, 5, 131-141.

${ }^{4}$ We thank Colleen Parks for raising this suggestion. 